\title{
Dynamics of Galaxies in Compact Groups I
}

\author{
C. Mendes de Oliveira
}

Instituto Astronômico e Geofísico, Universidade de São Paulo, Avenida Miguel Stéfano 4200, 04301-904 São Paulo, SP, Brazil

P. Amram

Observatoire de Marseille, 2 Place Le Verrier, 13248 Marseille, Cedex 04, France

\begin{abstract}
.
We describe a long-term program based on Fabry-Perot $\mathrm{H} \alpha$ velocity field data of compact groups taken at the ESO and the CFH $3.6 \mathrm{~m}$ telescopes. The main goals of our project are: 1) determine the evolutionary stages of the studied groups, 2) search for tidal dwarf galaxy candidates in interacting systems and 3 ) in combination with photometry available in the literature, determine the Tully-Fisher relation for the group galaxies. In this paper we summarize all the $\mathrm{H} \alpha$ Fabry-Perot data we have obtained to date and present a preliminary discussion of point 1).

The sample of 18 systems (with 64 galaxies) studied here contain groups at a variety of dynamical stages: from a false group that is in fact one single irregular galaxy with several star-forming blobs (e.g. HCG 18) to a group whose members are strongly interacting and possibly forming tidal dwarf galaxies (e.g. HCG 92) to a group in the final process of merging (e.g. HCG 31). A companion paper (Amram and Mendes de Oliveira, 2000) shows examples of galaxy velocity fields for groups in different dynamical stages.
\end{abstract}

\section{Introduction}

Anyone who has seen a picture of a compact group (CG) of galaxies (e.g. Stephan's quintet) will have asked the question: how could these galaxies be so close together? What is the fate of the dark halos? Are they stripped or do they merge together? If galaxies in compact groups are as close together in three-dimensions as they are in projection, their halos must overlap, merge or get stripped. This has fundamental consequences for the way galaxies will interact and merge (Barnes 1989). Indeed, compact groups of galaxies are considered the best laboratories to study the effects of interactions and merging on the structure and dynamics of galaxies. Moreover, compact groups can be thought of as nearby sites which mimic the higher density environments of the young universe.

The best-studied sample of compact groups is that of Hickson (1982, the Hickson compact groups of galaxies or HCG's). The sample of 92 compact 
groups with three or more members (382 galaxies in total) has complete photometric and redshift information (Hickson et al. 1992). The groups were significantly "expanded" as new faint members were discovered. Some groups with originally four members were found to have as many as 45 galaxies after addition of the faintest members (group HCG 62, Zabludoff and Mulchaey 1998). HI data (mostly unpublished) have been obtained for about one fifth of the Hickson sample (see Verdes-Montenegro et al., these proceedings) and they show a variety of group dynamics and morphologies. Diffuse X-ray emission was detected in 22 Hickson compact groups with a detection rate of $\sim 75 \%$ (Ponman et al. 1996). These clearly showed that a large fraction of the CGs are physical systems in the sense that they contain hot gas in a gravitational potential well. However, it is becoming clear that compact groups do not form a homogeneous class and instead CG catalogues may contain a mixture of systems of different nature and/or in several different stages of dynamical evolution. Groups that are elliptical-dominated, X-ray emitters tend to have a large number of additional faint members and are considered to be physical groups. On the other hand, the spiral-rich, low velocity dispersion, non-X-ray emitting groups, tend to have few faint members, if any, and so tend to be classified either as chance alignments (Mamon, these proceedings) or groups in the early stages of dynamical evolution. A number of spiral-rich HCGs with no X-ray emission and few faint members could indeed be misclassified as false groups if not for the many signs of interaction displayed by each member galaxy (e.g. Mendes de Oliveira et al. 1998). The detailed dynamics and morphological study of the spiral-rich groups in Hickson's catalogue tend to show that the member galaxies are well aware of the presence of their neighbors and therefore they cannot be mere chance alignments in looser environments.

A long-term program was initiated in 1995 to develop sensitive tests of determining compact group dynamical stages through the study of the detailed 2-D kinematics of the member galaxies. In this context, we started an observational program to obtain Fabry-Perot $\mathrm{H} \alpha$ velocity field data of high spatial and spectral resolution for a number of compact group galaxies. Warm gas is present in both early and late-type galaxies and can be used as a tracer of recent or on-going interactions. Although the warm gas component usually contributes only a small fraction of the total mass of a galaxy, it responds very quickly to gravitational perturbations and therefore allows a detailed study of the recent history of interactions/accretions of the system.

The sample of groups we have observed so far is highly biased towards spiral-rich groups. A subset of these data has already been presented in several publications: for HCG 16 (Mendes de Oliveira et al. 1998), HCG 18 (Plana et al. 1999, this proceedings, and Plana et al. 2000), HCG 90 (Plana et al. 1998), HCG 92 (Plana et al. 1999) and the group that contains the Cartwheel galaxy (Amram et al. 1998). In the present paper we summarize the whole database we have in hand for 64 galaxies in 18 compact groups. We also classify the compact groups into five classes according to the presence of kinematic peculiarities in the velocity fields of the member galaxies. More details about each class and examples are given in Amram and Mendes de Oliveira (2000). 


\section{Observations and Reductions}

The first set of observations was carried out with the Fabry-Perot (FP) instrument CIGALE mounted on the ESO 3.6m telescope in August 1995. CIGALE is composed of a focal reducer (bringing the original $\mathrm{f} / 8$ focal ratio of the Cassegrain focus to $\mathrm{f} / 2$ ), a scanning FP, a narrow band interference filter and an Image Photon Counting System detector. The spectral and spatial samplings were $\sim 16 \mathrm{~km} \mathrm{~s}^{-1}$ and $1^{\prime \prime}$ respectively and the free-spectral range of the FP instrument was $388 \mathrm{~km} \mathrm{~s}^{-1}$. The field of view was $\sim 3.8 \times 3.8$ arcmin.

The second set of observations was carried out at the Canada-France-Hawaii $3.6 \mathrm{~m}$ telescope in August 1996. Observations were made with the MOS focal reducer in FP mode, attached to the F/8 Cassegrain focus and a CCD detector. The spectral and spatial samplings were $\sim 11 \mathrm{~km} \mathrm{~s}^{-1}$ and $1^{\prime \prime}$ respectively and the free-spectral range of the FP instrument was $263 \mathrm{~km} \mathrm{~s}^{-1}$. The field of view was $\sim 7.2 \times 7.2$ arcmin.

For both datasets the interference filters were centered around $\mathrm{H} \alpha$ redshifted to the distance of the group and they typically had FWHM $<20 \AA$. The total exposure times were 2 hours in all cases.

Reduction of the data was performed using the CIGALE/ADHOC software (Boulesteix 1993). The data reduction procedure has been extensively described in previous papers (e.g. Amram et al. 1996).

The observed groups are listed in Table 1. Columns 1 and 2 give the identification of the galaxy, column 3 marks in which telescope the observation was made. Except for the Cartwheel galaxy the following four columns list data from Hickson (1993). Columns 4 and 5 list the morphological types and heliocentric radial velocities of the galaxies respectively. Column 6 lists the absolute magnitudes determined from the $\mathrm{B}_{T}$ asymptotic magnitude corrected for internal and external extinction. Column 7 lists the length of the major axis of the $\mu_{B}=25.0$ magnitude $\operatorname{arcsec}^{-2}$ isophote (Hickson 1993) in units of kpc. The galaxy distance used to derive the values in columns 6 and 7 were obtained from the Hubble law, from the velocities listed in column 5, assuming an $H_{0}$ of 75 $\mathrm{km} \mathrm{s}^{-1} \mathrm{Mpc}^{-1}$. Column 8 lists the photometric position angle of the major axis at $2.7 \mathrm{kpc}$ (for $H_{0}=75 \mathrm{~km} \mathrm{~s}^{-1} \mathrm{Mpc}^{-1}$ ), as given in Mendes de Oliveira (1992). Column 9 lists our determination of the position angle of the kinematic major axis of the galaxy, as given by an eye estimate of the axis of symmetry of the velocity field. Column 10 marks if the velocity field of the galaxy is regular or irregular, where regular stands for a similar velocity field to that observed for normal field galaxies of similar luminosities and irregular describes a disturbed, asymmetric, unusual velocity field, in a broad sense. Galaxies for which no $\mathrm{H} \alpha$ emission was detected are marked with a letter "N". Empty fields indicate that the data for the galaxy have not been analyzed or we do not have the information yet. This is the case for close to $25 \%$ of our sample.

\section{Results}

We describe below the status of the data and the results we have obtained so far for each observed system. 
Table 1. Parameters for observed compact-group galaxies

\begin{tabular}{|c|c|c|c|c|c|c|c|c|c|}
\hline $\mathrm{HCG}$ & $\mathbf{G}$ & Telescope ${ }^{a}$ & $T^{b}$ & $V_{r a d}^{b}$ & $\mathrm{M}_{\mathrm{B}}{ }^{b}$ & $D_{25}^{B} / 2^{b}$ & $P A^{c}$ & $P A$ & $V F$ \\
\hline & & & & $\left.\mathrm{ms}^{-1}\right)$ & & (kpc) & Phot & Kin & $\mathrm{R}, \mathrm{I}, \mathrm{N}$ \\
\hline \multirow[t]{4}{*}{01} & $\mathrm{a}$ & $\mathrm{CFH}$ & Sc & $1023^{4}$ & -21.3 & 24.9 & 39 & - & - \\
\hline & $b$ & $\mathrm{CFH}$ & $\mathrm{Im}$ & 10266 & -20.6 & 28.4 & 129 & - & - \\
\hline & $\mathrm{c}$ & $\mathrm{CFH}$ & EO & 10056 & -20.1 & 7.0 & 133 & - & - \\
\hline & $\mathrm{d}$ & $\mathrm{CFH}$ & So & 10120 & -19.2 & 5.6 & 23 & - & - \\
\hline \multirow[t]{4}{*}{07} & a & $\mathrm{CFH}$ & $\mathrm{Sb}$ & 4210 & -20.8 & 15.5 & 153 & - & - \\
\hline & $\vec{b}$ & $\mathrm{CFH}$ & SBO & 4238 & -20.0 & 11.0 & 176 & - & - \\
\hline & $c$ & CFH & SBC & 4366 & -21.2 & 14.8 & 129 & 135 & R. \\
\hline & $d$ & $\mathrm{CFH}$ & $\mathrm{SBC}$ & 4116 & -18.9 & 10.2 & 58 & - & - \\
\hline \multirow[t]{3}{*}{10} & $\mathrm{a}$ & CFH & $\mathrm{SBb}$ & 5148 & -21.6 & 34.9 & 69 & - & $\mathrm{R}$ \\
\hline & $c$ & $\mathrm{ESO}$ & Sc & 4660 & -19.9 & 16.7 & 31 & - & I \\
\hline & $d$ & CFH & Scd & 4620 & -19.3 & 8.7 & 151 & - & $\mathrm{R}$ \\
\hline \multirow[t]{4}{*}{16} & $\mathrm{a}$ & ESO & SBab & 4152 & -21.0 & 9.4 & 133 & 0 & $\mathrm{R}$ \\
\hline & $\mathrm{b}$ & ESO & Sab & 3977 & -20.4 & 12.7 & 74 & 86 & I \\
\hline & $\mathrm{c}$ & ESO & $\mathrm{Im}$ & 3851 & -20.5 & 7.8 & 93 & 115 & I \\
\hline & d & ESO & $\mathrm{Im}$ & 3847 & -20.1 & 10.2 & 84 & 80 & I \\
\hline \multirow[t]{3}{*}{18} & $\mathrm{~b}$ & $\mathrm{CFH}$ & $\mathrm{SOa}$ & 4082 & -18.8 & 13.2 & - & - & 1 \\
\hline & $\mathrm{c}$ & $\mathrm{CFH}$ & $\mathrm{Im}$ & 4143 & -18.1 & 6.1 & - & - & I \\
\hline & $d$ & $\mathrm{CFH}$ & I $\mathrm{m}$ & 4067 & -18.6 & 5.7 & $\ldots$ & - & I \\
\hline \multirow[t]{3}{*}{19} & $\mathrm{a}$ & ESO & E2 & 4279 & -19.8 & 7.2 & - & - & I \\
\hline & $b$ & ESO & Sed & 4210 & -18.5 & 6.6 & $\ldots$ & 98 & $\mathbf{R}$ \\
\hline & $\mathrm{c}$ & ESO & $\mathrm{Sdm}$ & - & - & - & - & - & $\mathbf{R}$ \\
\hline \multirow[t]{4}{*}{31} & $\mathrm{a}$ & ESO & $\mathrm{Sdm}$ & 4042 & -18.8 & 8.4 & 90 & - & 1 \\
\hline & b & ESO & $\mathrm{Sm}$ & 4171 & -19.4 & 7.0 & 37 & 45 & $\mathrm{R}$ \\
\hline & $\mathrm{c}$ & ESO & $\mathrm{Im}$ & 4068 & -21.2 & 4.8 & 23 & - & I \\
\hline & d & $\mathrm{ESO}$ & Sbc & 26900 & -20.5 & 9.4 & - & - & - \\
\hline \multirow[t]{5}{*}{79} & $\mathrm{a}$ & $\mathrm{CFH}$ & EO & 4292 & -19.4 & 13.9 & 67 & - & I \\
\hline & b & $\mathrm{CFH}$ & So & 4446 & -20.1 & 17.4 & 32 & - & - \\
\hline & $\mathrm{c}$ & $\mathrm{CFH}$ & So & 4146 & -19.0 & 10.8 & 72 & - & - \\
\hline & $\mathrm{d}$ & $\mathrm{CFH}$ & $\mathrm{Sdm}$ & 4503 & -18.0 & 8.2 & 0 & - & $\mathrm{R}$ \\
\hline & $\mathrm{e}$ & $\mathrm{CFH}$ & Scd & 19809 & -21.2 & 14.2 & - & & - \\
\hline \multirow[t]{4}{*}{87} & a & $\mathrm{ESO}$ & $\mathrm{Sbc}$ & 8694 & -21.4 & 22.4 & 58 & 55 & I \\
\hline & $b$ & ESO & So & 8972 & -20.3 & 10.1 & 41 & - & $N$ \\
\hline & c & ESO & $\mathrm{Sd}$ & 8920 & -20.4 & 12.3 & 77 & 85 & 1 \\
\hline & d & $\mathrm{ESO}$ & $\mathrm{Sd}$ & 10200 & -18.8 & 5.1 & - & - & - \\
\hline \multirow[t]{4}{*}{88} & $\mathbf{a}$ & $\mathrm{ESO}$ & $\mathrm{Sb}$ & 6033 & -21.4 & 17.6 & 124 & 130 & $\mathbf{R}$ \\
\hline & $\bar{b}$ & ESO & SBb & 6010 & -21.3 & 13.2 & 52 & 160 & $\mathbf{R}$ \\
\hline & $\mathbf{c}$ & $\mathrm{CFH}$ & $\mathrm{Sc}$ & 6083 & -20.7 & 10.9 & 81 & - & $\mathrm{R}$ \\
\hline & d & CFH & $\mathrm{Sc}$ & 6032 & -20.0 & 12.8 & 78 & - & $\mathrm{R}$ \\
\hline \multirow[t]{5}{*}{89} & a & $\mathrm{ESO}$ & $\mathrm{Sc}$ & 8850 & -21.3 & 16.6 & 43 & 50 & I \\
\hline & $\bar{b}$ & $\mathrm{ESO}$ & $S B C$ & 8985 & -20.5 & 12.1 & 38 & 155 & $\mathrm{R}$ \\
\hline & c & $\mathrm{ESO}$ & Scd & 8872 & -19.8 & 8.3 & 6 & 0 & $\mathrm{R}$ \\
\hline & d & $\mathrm{ESO}$ & $\mathrm{Sm}$ & 8857 & -19.1 & 4.6 & 95 & 78 & $\mathrm{R}$ \\
\hline & $e^{d}$ & - & - & - & - & -. & - & 90 & $\mathrm{R}$ \\
\hline 90 & $\mathrm{~b}$ & $\mathrm{ESO}$ & E0 & 2525 & -20.1 & 6.5 & 55 & 60 & I \\
\hline & $\mathrm{c}$ & $\mathrm{ESO}$ & E0 & 2696 & -20.1 & 6.4 & 91 & 40 & $\mathrm{R}$ \\
\hline & $\mathrm{d}$ & ESO & Im & 2778 & -20.0 & 12.4 & 90 & 75 & I \\
\hline 91 & $\mathrm{a}$ & $\mathrm{ESO}$ & $\mathrm{SBC}$ & 6832 & -22.2 & 19.1 & 53 & 2 & $\mathrm{R}$ \\
\hline & $\mathrm{c}$ & ESO & $\mathrm{Sc}$ & 7319 & -20.5 & 11.3 & 19 & 145 & $\mathrm{R}$ \\
\hline & d & ESO & SBO & 7195 & -19.9 & 6.2 & 91 & - & $\mathrm{N}$ \\
\hline $92^{e}$ & $\bar{b}$ & $\mathrm{CFH}$ & $\mathrm{Sbc}$ & 5774 & -21.3 & 24.9 & 16 & - & $\mathrm{I}$ \\
\hline & $\mathrm{c}$ & $\mathrm{CFH}$ & $\mathrm{SBC}$ & 6764 & -21.5 & 23.1 & 139 & - & I \\
\hline & d & $\mathrm{CFH}$ & $\mathrm{Sc}$ & 6630 & -21.1 & 15.7 & 99 & - & $\mathrm{N}$ \\
\hline 93 & $\mathrm{a}$ & $\mathrm{CFH}$ & E1 & 5140 & -21.6 & 12.0 & 157 & - & - \\
\hline & c & CFH & $S \mathbf{B a}$ & 5132 & -20.2 & 11.9 & 91 & - & - \\
\hline 96 & $\mathrm{a}$ & $\mathrm{ESO}$ & $\mathrm{Sc}$ & 8698 & -21.8 & 18.7 & 98 & 125 & I \\
\hline & $b$ & ESO & $\mathrm{E} 2$ & 8616 & -20.8 & 10.4 & 36 & - & $\mathrm{N}$ \\
\hline & c & ESO & $\mathrm{Sa}$ & 8753 & -19.7 & 6.9 & 29 & 45 & $\mathrm{R}$ \\
\hline & d & ESO & $\mathrm{Im}$ & 8975 & -18.8 & 3.8 & 170 & 190 & I \\
\hline 100 & a & both & $\mathrm{Sb}$ & 5300 & -20.6 & 11.4 & 77 & 72 & I \\
\hline & $\mathrm{b}$ & both & $\mathrm{S}_{\mathrm{m}}$ & 5253 & -19.3 & 5.9 & 164 & 135 & I \\
\hline & c & $\mathrm{CFH}$ & $\mathrm{SBc}$ & 5461 & -19.1 & 7.8 & 67 & - & - \\
\hline & $\mathrm{d}$ & $\mathrm{CFH}$ & Scd & - & & - & - & - & - \\
\hline Cartwheel & $\mathrm{a}$ & ESO & Pec & 9050 & -21.5 & - & - & 126 & I \\
\hline & $\mathbf{b}$ & ESO & $\mathrm{b}$ & 8657 & -19.6 & - & - & 223 & I \\
\hline
\end{tabular}

a Telescope used, either the ESO or CFH $3.6 \mathrm{~m}$ telescope; ${ }^{b}$ from Hickson et al. $1992\left(H_{0}=75\right.$ $\left.\mathrm{km} \mathrm{s}^{-1} \mathrm{Mpc}^{-1}\right) ;{ }^{c}$ from Mendes de Oliveira 1992; ${ }^{d}$ dwarf galaxy south of HCG 89a; not in Hickson's catalogue; ${ }^{e}$ Stephan's quintet; observed also at the Russian $6 \mathrm{~m}$ telescope 
HCG 01: This is the most distant group of our sample, at $z=0.0339$. All catalogued members were observed. H01a and $\mathrm{H} 01 \mathrm{~b}$ show strong central $\mathrm{H} \alpha$ in emission while H01c and H01d present weak emission. Two bright stars in the field affected the observations of H01c. Reduction of the data for this group is not complete.

HCG 07: Galaxy HCG 07c has a perfectly symmetric velocity field and rotation curve. The other three members have small amounts of gas. Reduction of the data for this group is not complete.

HCG 10: Three of the four group galaxies were observed. Galaxy 10a (of morphological type $\mathrm{SBb}$ ) has little $\mathrm{H} \alpha$ gas, concentrated in the central region. HCG 10c has a peculiar velocity field, with the possible presence of a second component while HCG $10 \mathrm{~d}$ has a normal rotation curve. HCG $10 \mathrm{~b}$ (an elliptical galaxy) was not observed.

HCG 16: The observations for this group cover the four core galaxies HCG $16 \mathrm{a}, \mathrm{b}, \mathrm{c}$ and $\mathrm{d}$. The velocity fields show kinematic peculiarities for three of the four galaxies: HCG 16b, c and d. Fig. 1 shows the monochromatic images and velocity fields for galaxies $\mathrm{HCG} 16 \mathrm{c}$ and $\mathrm{d}$. Misalignments between the kinematic and photometric axes of gas and stellar components (HCG 16b,c,d), double gas systems (HCG 16c) and severe warping of the kinematic major axis (HCG 16b and c) were some of the peculiarities detected. Major merger events have possibly taken place in at least two of the galaxies of the group, HCG 16c and d, based on their significant kinematic peculiarities, their double nuclei and high infrared luminosities. Galaxy HCG 16b, an Sb galaxy, also presents some of the kinematic evidences for past accretion events. Its gas content, however, is very sparse, limiting our ability to find other kinematic merger indicators, if they are present. We find that the merger remnants in the compact group HCG 16 have significantly smoother optical profiles than isolated mergers, i.e., they show an amorphous morphology and no signs of tidal tails. Tidal arms and tails formed during the mergers may have been shredded by subsequent interactions in the group (Barnes and Hernquist 1992) or alternatively they may have never been formed. The velocity field of the galaxy HCG 16 a shows grand-design isovelocity lines with no signs of disturbances inside a radius of $\sim R_{25}$. The normality of the velocity field suggests that this galaxy may be a fairly recent acquisition of the compact group. Our observations suggest that HCG 16 may be a young compact group in formation through the merging of close-by objects in a dense environment. These results were described in detail in Mendes de Oliveira et al. (1998)

HCG 18: This quartet is formed by an accordant triplet (HCG 18b, c and d) and one interloper (HCG 18a) at a much higher redshift. Only the three low-redshift objects were observed. HCG $18 \mathrm{~b}, \mathrm{c}$ and d do not show independent motion but instead a complex common velocity field that involves the three galaxies as if they were one body (see Plana et al., these proceedings). The $\mathrm{H} \alpha$ data confirms an earlier result obtained from an HI study of the group (Williams \& van Gorkom 1988) that HCG 18 is a single irregular galaxy with clumps of 

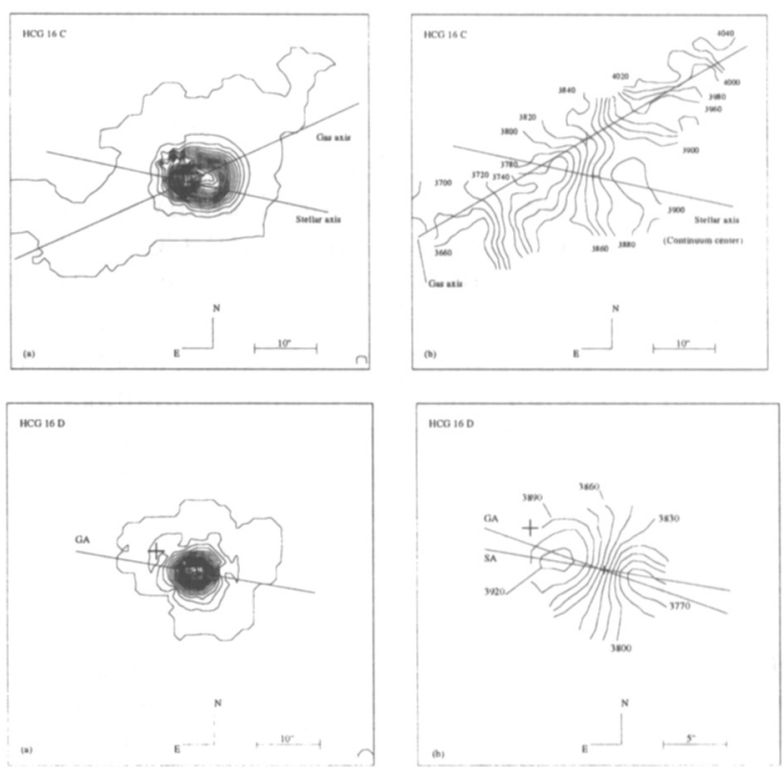

Figure 1. $\mathrm{H} \alpha$ images and velocity fields of $\mathrm{HCG} 16 \mathrm{c}$ and $\mathrm{HCG} 16 \mathrm{~d}$.

star formation coinciding with what was catalogued by Hickson (1982) as distinct galaxies. Details of this work are described in Plana et al. (2000).

HCG 19: Galaxy HCG 19a is an elliptical galaxy with an extended gas disk reaching a radius of 10 arcsec. Galaxies $\mathrm{b}$ and $\mathrm{c}$ have normal velocity fields and rotation curves.

HCG 31: This is the group with the most disturbed galaxies. HCG 31a and HCG 31c are a clear case of an ongoing merger of two or more nuclei with the possible presence of several candidate tidal dwarf galaxies formed in the merger (east and south of the system). HCG 31b has a regular velocity field for its Sm type.

HCG 79: Seyfert's Sextet is one of the most compact groups in Hickson's sample. It is in reality a quartet since one of the galaxies is an interloper with much higher redshift and the sixth member (east of HCG 79b) is indeed not a galaxy but a low-surface brightness extension of HCG $79 \mathrm{~b}$. Emission is detected in all accordant galaxies. HCG 79a, an elliptical galaxy, has a strong dust lane along its major axis. The group contains only one spiral galaxy (HCG 79d). It has a fairly regular velocity field for its luminosity and type.

HCG 87: Three galaxies of this quartet were observed (the fourth one was outside of the filter range). HCG $87 \mathrm{a}$, a peanut-shape galaxy (Mihos et al. 1995), has strong dust lanes aligned with the major axis of the galaxy. This 
group has been recently imaged by HST. Reduction of the data for this group is not complete.

HCG 88: This quartet is one of the groups with lowest projected velocity dispersions ( $\left.\sigma=27 \mathrm{~km} \mathrm{~s}^{-1}\right)$ in Hickson's catalogue. All four galaxies were observed in $\mathrm{H} \alpha$ and the velocity fields and rotation curves were found to be normal in all cases. Rubin et al. (1991) observed that HCG 88a has non-coinciding shapes for the receding and approaching sides. If we use their same PA and inclination and simulate a slit, we reproduce their result. However, with the information from the 2-D map, a more precise PA and inclination from the kinematics give a normal profile for this galaxy. Similar findings for HCG 16a and HCG 100a were pointed out by Rubin et al. (1991) but again our observations show that both sides of the rotation curves are coincident. For HCG $88 \mathrm{~b}$, the signature of the bar and spiral arms are clearly seen on the velocity field, which is regular for its type and luminosity. Galaxies HCG 88c and HCG 88d have normal velocity fields.

HCG 89: Group HCG 89 is one of the most distant groups from our sample $(z=0.0297)$. The four galaxies catalogued by Hickson were observed in $\mathrm{H} \alpha$ as well as a new dwarf galaxy south of HCG 89a. The velocity maps of all members, with exception of HCG $89 \mathrm{a}$, which has an irregularity in its center, have normal shapes.

HCG 90: Mapping of the gas kinematics was possible to $\sim 2 \mathrm{r}_{\text {eff }}$ for the disk galaxy H90d and to $\sim 1.3 \mathrm{r}_{\text {eff }}$ and $\sim 1.7 \mathrm{r}_{\text {eff }}$ for the early-type galaxies $\mathrm{H} 90 \mathrm{~b}$ and H90c. Evidence for ongoing interaction was found in the properties of the warm gas of the three galaxies. The system H90bd which was previously suspected to be an optical double may in reality be a system in interaction. In the region where the galaxies spatially overlap in projection (their continuum centers are only $25^{\prime \prime}$ apart), the gas profiles are separated in velocity space by $\sim 50-100$ $\mathrm{km} \mathrm{s}^{-1}$. The gas component of the early-type galaxy is highly concentrated in the region closest to the irregular galaxy, where the interaction between the two galaxies may be taking place. The velocity fields of these galaxies are disturbed, most probably due to the ongoing interaction; they indicate that the galaxies are in pro-grade orbits, which is a favorable condition for merging. These results were described in detail in Plana et al. (1998)

HCG 91: Three of the galaxies in this quartet were observed (H91a, c and d). Two of them (H91a and c) contain emission and their velocity fields are normal.

HCG 92: Galaxies H92b, c and d were observed. The $\mathrm{H} \alpha$ observations showed an intragroup arclike feature formed of bright $\mathrm{H} \alpha$ knots and diffuse gas distributed along tidal tails associated with $\mathrm{H} 92 \mathrm{~b}$ and possibly also H92d (Plana et al. 1999). We found kinematic and photometric evidence for the presence of five candidate tidal dwarf galaxies in Stephan's quintet formed out of material thrown in the intergalactic medium during galactic collisions. The central regions of the two most probable parent galaxies, H92b and $\mathrm{H} 92 \mathrm{~d}$, contain lit- 
tle or no gas whereas the intragroup medium, and particularly the optical tails that seem to be associated with $\mathrm{H} 92 \mathrm{~b}$ are rich in ionized gas and they contain a number of giant $\mathrm{H}$ Ir regions. H92c has a small, centrally concentrated warm gas reservoir.

HCG 93: Two of the five accordant members of this group were observed (H93a,c). Reduction of the data is not complete.

HCG 96: All group galaxies were observed. Except for the E2 galaxy H96b, all others have emission. Galaxies $96 \mathrm{a}$ and $96 \mathrm{~d}$ have slightly peculiar velocity fields.

HCG 100: All four galaxies of the group were observed and all of them have emission. HCG 100a has centrally concentrated emission, with a peculiar rotation curve. HCG $100 \mathrm{~b}$ has a large misalignment between the photometric and kinematic major axis. Reduction of the data for this group is not complete.

Cartwheel group: Three galaxies at a redshift of $\sim 0.03$ were observed in the field of the Carthweel galaxy. Velocity fields for two galaxies were measured, for the main galaxy of the system, the Cartwheel galaxy, and for a close spiral companion. The early-type companion contained no gas. The velocity field and the rotation curve of the spiral companion showed classical differential rotation. We found that the Cartwheel galaxy contains strong $\mathrm{H} \alpha$ in emission asymmetrically distributed along its outer ring, with some faint emission detected inside it. The velocity field and the rotation curve of the Cartwheel galaxy is clearly dominated by differential rotation although second-order effects cause distortions which can be understood in a scenario where expansion and density-wave motions are also considered. Our best model-fit to the observed velocity field of the Cartwheel galaxy includes three components for the kinematics of its outer ring: a circular velocity of $217 \mathrm{~km} \mathrm{~s}^{-1}$, an expansion velocity of $13-30 \pm 10 \mathrm{~km} \mathrm{~s}^{-1}$ and a sinusoidal perturbation with an amplitude of $20 \pm 5 \mathrm{~km} \mathrm{~s}^{-1}$. This expansion velocity, lower than found in previous studies, implies that the ring could be older than previously thought. The inner rotation curve of the Cartwheel galaxy is consistent with a progenitor which had the morphology of a late-type spiral galaxy. These results were described in detail in Amram et al. (1998).

\section{Discussion}

The 18 groups we have observed so far present general similarities but also many individualities. In the following we attempt to describe and exemplify five classes of compact groups found in our sample. We note, however, that we may not have covered all possible dynamical stages of compact groups since our sample is highly biased towards spiral-rich groups. There is a lack of elliptical-rich groups which are indeed the ones that have most often been identified in X-rays and are the ones where there is no doubt about their physical nature as a bound system. We may, therefore, be missing systems which are in a different stage of evolution than what is listed below. 
Merging groups - Egs: HCG 31 and HCG 79. These are systems probably in the last stage of evolution before they become a single merged galaxy. They have very disturbed gas dynamics and in the optical they present a halo of light involving all galaxies of the group. They contain galaxies that are small in size compared to other galaxies of similar luminosities. In the case of HCG 79, for example, the optical diameter of the whole group is only $\sim 25 \mathrm{kpc}\left(H_{0}=75\right.$ $\mathrm{km} \mathrm{s}^{-1} \mathrm{Mpc}^{-2}$ ). The galaxies have already lost or are about to lose their identity.

Strongly interacting groups - Egs: HCG 16, HCG 90, HCG 92, HCG 100. These systems present evidence for strong interaction and merging but the galaxies clearly maintain their identity. In H92 the warm gas is not attached to the galaxies but to the tidal features. In HCG 16, there is evidence that three of the galaxies are the result of merging events. H90 has a halo of light around three of the member galaxies and has a bridge of warm gas between two of the systems.

Mildly interacting groups - Egs: HCG 10, HCG 19, HCG 89, HCG 96. These are systems whose gas properties present mild evidence for interaction. At least one of the galaxies presents a disturbed rotation curve.

Kinematically undisturbed groups - Eg: HCG 88. None of the galaxies present kinematic peculiarities as measured from the velocity field of the warm gas. This may be a group in the first stages of evolution. It is interesting to note that H88 was classified as an "isolated" and "real" compact group by Ribeiro et al. (1998), since it has one of the poorest neighborhoods of all groups in Hickson's catalogue.

False group, system is in reality a single galaxy - Eg: HCG 18 - All members in the group present a single velocity pattern. Systems which were originally catalogued as individual galaxies are in fact blobs of star formation inside a single galaxy. The HI properties of this system has been studied in detail confirming the hypothesis that the system may indeed be one single irregular galaxy.

\section{Conclusion}

Our study shows that the Hickson compact group catalogue may contain a variety of objects in several different stages of dynamical evolution. We find that for a subsample of spiral-rich dominated groups these can range from a false group that is in fact one single irregular galaxy with several star-forming blobs to undisturbed groups, groups with evidence for mild and strong interactions and groups in the final process of merging.

Acknowledgments. We would like to thank the meeting organizers for the invitation. We would also like to thank our collaborators Henri Plana, Chantal Balkowski, Jacques Boulesteix and Michael Bolte for their invaluable contribution to this project. 


\section{References}

Amram, P., Balkowski, C., Boulesteix, J., Cayatte, V., Marcelin, M., Sullivan, W.T. III 1996, A\&A, 310, 737

Amram, P., Mendes de Oliveira, C. 2000, in Galaxy Dynamics: from the Early Universe to the Present, eds. F. Combes, G.A. Mamon and V. Charmandaris, ASP Conference Series, in press.

Amram, P., Mendes de Oliveira, C., Boulesteix, J., Balkowski, C. 1998, A\&A, 330,881

Barnes, J. 1989, Nature, 338, 123

Barnes, J., Hernquist, L. 1992, ARA\&A, 30, 705

Boulesteix, J. 1993, "ADHOC reference manual", Publications de l'Observatoire de Marseille.

Hickson, P., 1982, ApJ, 255, 382

Hickson, P., Mendes de Oliveira, C., Huchra, J.P., Palumbo, G.G.C. 1992, ApJ, 399,353

Hickson, P. 1993, Astrophys. Letters \& Communications Vol. 29 numbers 1-3

Mendes de Oliveira, C. 1992, PhD Thesis, U. of British Columbia

Mendes de Oliveira, C., Plana, H., Amram, P., Bolte, M., Boulesteix, J. 1998, ApJ, 507, 691

Mihos, J. C., Walker, I. R., Hernquist, L., Mendes de Oliveira, C. and Bolte, M. 1995, ApJ, 477, L87

Plana, H., Amram, P., Mendes de Oliveira, C., Balkowski, C. 2000, in preparation

Plana, H., Mendes de Oliveira, C., Amram, P., Balkowski, C. 1999, Apj, 516, L69

Plana, H., Mendes de Oliveira, C., Amram, P., Boulesteix, J. 1998, AJ, 116, 2123

Ribeiro, A., de Carvalho, R., Capelato, H. V., Zepf, S., 1998, ApJ, 497, 72

Rubin, V. C., Hunter, D. A., Ford, K. W. 1991, ApJS, 76, 153

Williams, B. A., van Gorkom, J. H. 1988, AJ, 95, 352

Zabludoff, A., Mulchaey, J. 1998, ApJ, 496, 39 